\title{
Meningococcal genomics: two steps forward, one step back.
}

\author{
Signature tagged mutagenesis of $\mathrm{N}$. meningitidis has identified genes that are required for septicemic infection. \\ However, these high-tech studies also raise questions about how to choose the isolates of pathogens that will yield \\ the most useful information (pages 1269-1274).
}

NFECTION WITH THE human pathogenic bacterium Neisseria meningitidis causes bacteremia and meningitis, and in many European and American countries leads to more death and disability among infants than any other microbial infection $^{1}$. In Asia and Africa, pandemics of meningococcal disease also cause high levels of morbidity and mortality. This is in part due to the lack of vaccines against this microorganism-the development and implementation of effective and safe meningococcal vaccines remains an urgent priority throughout the world. Asin other areas of medicine, the application of genomic technology to meningococcal disease has generated optimism that a new era of understanding is dawning, typified by the completion earlier this year of the genome sequences of two distinct meningococci ${ }^{2,3}$. In this issue, Sun et al. ${ }^{4}$ report the use of signature tagged mutagenesis (STM) to identify genes essential for bacteremia (bacterial infection of the blood) the prerequisite to the pathological complications of meningococcal infection.

The STM approach is based on genomic mutagenesis using transposon donors labelled with unique nucleotide sequences (tags). STM is a powerful tool useful for identifying genes involved in pathogenesis in a variety of animal models $s^{5}$, and allows a large numbers of mutants to be analyzed simultaneously in vivo. The novelty of the work described by Sun et al. ${ }^{4}$ is that the insertional mutagenesis was carried out in vitro, overcoming limitations imposed by the currently available tools for the gen etic manipulation of $\mathrm{N}$. meningitidis. The clever twist is that the authors were able to exploit the transformation and recombination systems of this bacterium to complete the construction of the mutant library. After screening a library of 2,850 insertional mutants, the authors identified 73 genes putatively involved in septiceamic growth, of which only eight have previously been associated with pathogenicity $^{4}$.

Genomic studies are substantial investments in time and money, yet they have potential to produce new resources and

\section{MARTIN C. MAIDEN ${ }^{1} \&$ IAN M. FEAVERS ${ }^{2}$}

ideas for future research, as well as to influence other areas of research. This is simultaneously an opportunity and a challenge. Genomic studies generate

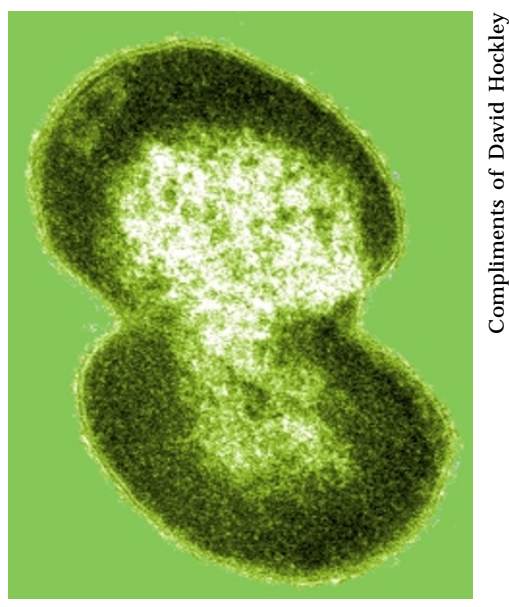

Neisseria meningitidis

large quantities of data, which in most instances is considered to be a good thing. However, the amount of resources required to perform such studies reminds us that we must carefully think them through before they are initiated. Once a genomic or functional genomic study has commenced, many important factors cannot changed, such as the organism whose DNA is chosen for analysis. This is of particular concern in the case of bacteria, for which it is all too easy to overlook the fact that all bacteria are not created equal.

Like most microorganisms, there are numerous genetic and antigenic variants of $\mathrm{N}$. meningitidis. These have been identified by population studies employing techniques such as multi-locus enzyme electrophoresis ${ }^{6}$ and lateral multi-locus sequence typing ${ }^{7}$. We know that some meningococcal lineages cause disease often (the hyperinvasive lineages), some lineages cause more severe forms of disease than others (described as hypervirulent), and others rarely cause disease at all. The situation is further complicated by the fact that meningococcal antigens are able to move among genetic lineages, presumably at the dictates of immune selection ${ }^{8}$. A number of meningococci of known lineages have been extensively used in vaccine development, notably the isolate known as $\mathrm{H} 44 / 76$, which was isolated in Norway during a hyperendemic outbreak in the 1970s (ref. 9). A different variant of the same bacterial lineage was used in a vaccine developed in $\mathrm{Cuba}^{10}$.

However, genomic studies are not usually based on these past data and insights. For example, the isolate chosen for the study of Sun et al. ${ }^{4}$, C311, is relatively obscure and has not been widely used in other studies of meningococcal biology. It is not a well characterized representative variant of a meningococcal hyperinvasive lineage, it has not been used in vaccine development, and it is not one of the isolates for which the complete genome has been determined. To a degree, these facts compromise the value of the results of the study for the community as a whole.

Clearly, if we are to maximise the benefits from the investment in meningococcal genomics (or any other pathogen), there needs to be a consensus on which isolates should be regarded as type-strains and these strains should form the basis of fundamental research. They need to be chosen with care by the community as a whole and not by individual researchers. Definitive cultures of the isolates need to be deposited in culture collections, made widely available and the unofficial distribution of cultures discouraged for fear of generating variants. Once upon a time these ideas were basic ten ets of microbiology.

Genomic studies are a welcome and invaluable addition to the biomedical research arsenal, and these results represent an important resource for future work on meningococcal disease. However, researchers must also carefully consider basic biology before initiating these types of high-tech experiments. It is perhaps an appropriate time to ask the questions of how this technological 
potential is to be most effectively and efficiently harnessed in the fight against infectious diseases.

1. Schwartz, B., Moore, P.S. \& Broome, C.V. Global epidemiology of meningococcal disease. Clin. Microbiol. Revs 2, s118-s124 (1989).

2. Tettelin, H. et al. Complete Genome Sequence of Neisseria meningitidis Serogroup B Strain MC58. Science 287, 1809-1815 (2000).

3. Parkhill, J. et al. Complete DNA sequence of a serogroup A strain of Neisseria meningitidis Z2491. Nature 404, 502-506 (2000).

4. Sun, Y.-H., Bakshi, S., Chalmers, R., \& Tang, C.M Functional genomics of Neisseria meningitides pathogenesis. Nature Med. 6, 1269-1274 (2000).
5. Hensel, M. et al. Simultaneous identification of bacterial virulence genes by negative selection. Science 269, 400-403 (1995)

6. Caugant, D.A. et al. Genetic structure of Neisseria meningitidis populations in relation to serogroup, serotype, and outer membrane protein pattern. J. Bacteriol. 169, 2781-2792 (1987).

7. Maiden, M.C.J. et al. Multilocus sequence typing: a portable approach to the identification of clones within populations of pathogenic microorganisms. Proc. Natl. Acad. Sci. USA. 95, 3140-3145 (1998).

8. Gupta, S. et al. The maintenance of strain structure in populations of recombining infectious agents. Nature Med. 2, 437-442 (1996).

9. Bjune, G. et al. Effect of outer membrane vesicle vaccine against group B meningococcal disease in Norway. Lancet 338, 1093-1096 (1991).
10. de Moraes, J.C. et al. Protective efficacy of a serogroup B meningococcal vaccine in Sao Paulo, Brazil. Lancet 340, 1074-1078 (1992).

${ }^{1}$ W ellcome Trust Centre for the Epidemiology of Infectious Diseases, Department of Zoology University of Oxford, South Parks Road, Oxford OX1 3PS. United Kingdom.

${ }^{2}$ Division of Bacteriology, National Institute for Biological Standards and Control, Blanche Lane, South Mimms, Potters Bar, EN6 3QG.

United Kingdom.

Email: ifeavers@nibsc.ac.uk

\title{
Not a minute to waste
}

\begin{abstract}
We are finally beginning to unlock the mechanisms underlying $\mathrm{Ca}^{2+}$-stimulated muscle differentiation and cytokinemediated muscle wasting. Gaining a better understanding of the signaling pathways that regulate muscle development and decay improves the prospects for repairing aged, injured and diseased muscle.
\end{abstract}

$\mathrm{E}^{\mathrm{w}}$ LUCIDATION OF THE mechanisms that regulate muscle regeneration, decay and hypertrophy provides the cornerstone for therapeutic intervention into musclerelated diseases. These processes are adaptive responses to physical, injury-related and pathological stimuli. Age and woundinduced muscle wasting, cachexia, and myopathies reflect malfunction in the bal ance between regeneration and catabolism. Muscle wasting is observed in $20-80 \%$ of cancer patients and also in people with chronic diseases ${ }^{1}$. The impact of muscle wasting on therapeutic tolerance, morbidity and mortal ity in AIDS and cancer patients underscores the importance of understanding the process of differentiation, regeneration and decay $^{2,3}$.

Two recent studies exploiting an in vitro model of differentiation have revealed the mechanisms underlying the regulation of muscle differentiation and decay. In the 2 November issue of Nature, McKinsey et al. ${ }^{4}$ report that $\mathrm{Ca}^{2+}$ dependent regulation of a chromatin remodelling enzyme regulates muscle differentiation, while in the 29 September issue of Science, Guttridge et al. ${ }^{5}$ report that NF- $\kappa B$ plays a role in cytokine-induced muscle wasting and dysfunction.

Great strides have been made in improving our understanding of muscle differentiation (myogenesis), hypertrophy, regeneration, and decay through the exploita-

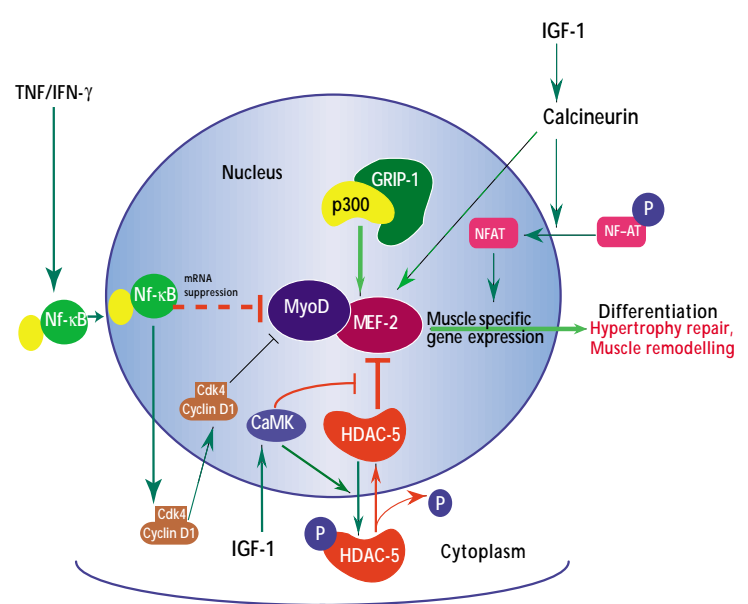

Fig. 1 Signal transduction pathways that regulate myogenesis. The cooperative interaction of the transcription factors MyoD and MEF2, along with the co-activators p300 and GRIP-1, activate muscle-specific transcription and myogenesis. Cyclin D1 mediates translocation of Cdk4 to the nucleus, repressing MyoD function and myogenesis. The histone deacetylase HDAC-5, upon dephosphorylation and translocation to the nucleus, directly inhibits MEF2, preventing myogenesis. McKinsey et al. ${ }^{4}$ report that calcium/calmodulin-dependent kinase (CaMK), stimulates myogenesis and prevents formation of MEF2/HDAC complexes by inducing phosphorylation and nuclear export of HDACs 4 and 5 . CaMK is activated by insulin-like growth factor-1 (IGF-1). IGF-1 also activates myogenesis through a calcineurin and NF-AT mediated signaling pathway. Guttridge et al. ${ }^{5}$ report that cytokines such as tumor necrosis factor- $\alpha$ (TNF- $\alpha$ ) and interferon- $\gamma$ (IFN- $\gamma$ ) induce the activation and nuclear translocation of NF- $\mathrm{KB}$, which suppresses myoD mRNA, leading to dysfunction of skeletal myofibers. Induction of muscle differentiation may be useful in reversing muscle hypertrophy and inducing muscle remodelling. multinucleated myotubes that acquire a muscle-specific phenotype. This cellular morphogenesis is accompanied by musclespecific gene expression.

A group of basic-helix-loop-helix proeins encoded by the myoD gene family (myoD, myf-5, myogenin, and MRF-4) and a second class of transcription factors, the myocyte enhancer factor2 family (MEF2A-D), are required for proper muscle differentiation. The MyoD and MEF2 transcription factors function in a cooperative manner to control the mutually exclusive events of division and differentiation?.

Transcription is also regulated by the structural conformation of chromatin, a complex made up of DNA, histones and other proteins. Chromatin structure is regulated by cofactors such as histone acetyltransferases (HATs) and deacetylases (HDACs). Histone acetylation and deacetylation affect accessibility of DNA to the transcriptional machinery, leading to transcriptional activation or repression, respectively. Both histone hyperacetylation and hypoacetylation have been associated with the neoplastic process ${ }^{8}$.

The cofactors GRIP-1, CBP/p300 and PCAF have HAT activity and function as co-activators for MEF2C during myogenesis ${ }^{9-10}$. Class II HDACs (HDAC-4 and -5) interact with MEF2, inhibiting its ability to activate transcription ${ }^{11}$ and myoblast differentiation ${ }^{12}$. The ability of class II HDACs to repress tran- 